\title{
Thioridazine, an antipsychotic drug, elicits potent antitumor effects in gastric cancer
}

\author{
JIASHENG MU ${ }^{1,2^{*}}$, HAINENG XU ${ }^{3 *}$, YU YANG $^{3}$, WEIDAN HUANG ${ }^{3}$, JING XIAO $^{4}$, \\ MAOLAN LI ${ }^{1,2}$, ZHUJUN TAN ${ }^{1,2}$, QICHEN DING ${ }^{1,2}$, LIN ZHANG $^{1,2}$, \\ JIANHUA LU ${ }^{1,2}$, XIANGSONG WU ${ }^{1,2}$ and YINGBIN LIU ${ }^{1,2}$ \\ ${ }^{1}$ Department of General Surgery and Laboratory of General Surgery, Xinhua Hospital, Affiliated to \\ Shanghai Jiao Tong University School of Medicine, Shanghai 200092; ${ }^{2}$ Institute of Biliary Tract Disease, \\ Shanghai Jiao Tong University School of Medicine, Shanghai 200092; ${ }^{3}$ State Key Laboratory of Cell Biology, \\ Institute of Biochemistry and Cell Biology, Shanghai Institutes for Biological Sciences, Chinese Academy of Sciences, \\ Shanghai 200031; ${ }^{4}$ College of Life Science, Henan Normal University, Xinxiang, Henan 453007, P.R. China
}

Received January 10, 2014; Accepted February 13, 2014

DOI: 10.3892/or.2014.3068

\begin{abstract}
Thioridazine, an antipsychotic drug, has been reported to induce apoptosis in various types of cancer cells, with specificity on targeting cancer stem cells (CSCs). However, whether it elicits anticancer effects in gastric cancer has never been reported. In the present study, we examined the ability of thioridazine to induce cell death in the gastric cancer cell lines NCI-N87 and AGS, and detected its in vivo tumor inhibition capacity. Thioridazine elicited cytotoxic effects on NCI-N87 and AGS cells in a dose-dependent manner, and inhibited the colony formation abilitiy of the NCI-N87 and AGS cells. Thioridazine treatment induced nuclear fragmentation, increased the proportion of sub-G1 phase cells, and elevated the percentage of Annexin V-positive cells, suggesting the occurrence of apoptosis. Moreover, thioridazine induced gastric cancer cell apoptosis in a caspase-dependent manner, as shown by a decrease in the precursors of casapse- 9 , caspase- 8 and caspase- 3 , and by the ability of the caspase inhibitor Z-VAD-FMK to reverse the cytotoxic effect of thioridazine. JC-1 staining further revealed
\end{abstract}

Correspondence to: Dr Yingbin Liu, Department of General Surgery, Xinhua Hospital, Affiliated to Shanghai Jiao Tong University, School of Medicine, 1665 Kong-Jiang Road, Shanghai 200092, P.R. China

E-mail: liuybphd@126.com

Dr Haineng Xu, State Key Laboratory of Cell Biology, Institute of Biochemistry and Cell Biology, Shanghai Institutes for Biological Sciences, Chinese Academy of Sciences, 320 Yue-Yang Road, Shanghai 200031, P.R. China

E-mail: xuhaineng123@163.com

*Contributed equally

Key words: thioridazine, antipsychotic drug, gastric cancer, apoptosis, caspase, mitochondria, in vivo that thioridazine induced gastric cancer cell apoptosis via the mitochondrial pathway. In addition, thioridazine pretreatment inhibited the growth of NCI-N87 cell-derived tumors. The present study demonstrated that the antipsychotic drug thioridazine possesses anti-gastric cancer ability through in vitro and in vivo experiments, suggesting thioridazine as a potential drug in gastric cancer therapy.

\section{Introduction}

Gastric cancer is the fourth most common cancer worldwide with 989,600 new cases diagnosed in 2008, and it is the second leading cause of cancer-related death $(1,2)$. Gastric cancer is either asymptomatic or causes non-specific symptoms at an early stage, and thus the majority of patients present with advanced stage disease at the time of initial diagnosis, leading to the poor prognosis of gastric cancer. Gastric cancer treatment involves surgery, chemotherapy, radiation therapy and their combinations. However, current drugs are confronted with low efficacy due to the high rate of patients at the advanced stage of gastric cancer. Thus, the development of novel effective drugs for gastric cancer therapy is urgently needed.

Thioridazine, an antagonist of the dopamine receptor D2 family proteins, was initially developed as an antipsychotic drug, and recently its anticancer function was revealed. Studies have revealed the antiproliferative and apoptosis induction capacities of thioridazine in neuroblastoma, glioma, leukemia, breast cancer, cervical cancer and endometrial cancer (3-6). A gene signature-based approach identified thioridazine as an inhibitor of PI3K/Akt signaling in ovarian cancer cells. It downregulated cell cycle regulators cyclin D1 and CDK4, and upregulated p21, p16 and p-CDC25A, leading to the G0/ G1 phase arrest of the cells (7). Research on cervical and endometrial cancer cells disclosing the involvement of the $\mathrm{PI} 3 \mathrm{~K} / \mathrm{Akt} / \mathrm{mTOR}$ pathway in thioridazine-induced apoptosis further supported this finding (3). Thioridazine was also found to exhibit an anti-angiogenic effect through the FAK/mTOR pathway (8), which may act in inhibiting tumor growth in vivo. 
In addition, Byun et al (8) found that thioridazine inhibited cancer cell growth while sparing various types of normal cells (6).

Chemoresistance is one of the limitations to gastric cancer therapy and this issue must be remedied. Research has revealed the capacity of thioridazine to reverse the chemoresistance of cancer cells. Thioridazine was found to overcome the resistance of P388 murine leukaemia cells to doxorubicin (9). Thioridazine was also able to reverse the chemoresistance of human KB carcinoma cells by increasing the accumulation of chemotherapeutic drugs doxorubicin, vinblastine and dactinomycin in the cells (10). Thioridazine in combination with verapamil exhibited enhanced ability to reverse the multidrug resistance of cancer cells (11). Moreover, thioridazine was found to inhibit the expression of P-glycoprotein and induce the apoptosis of ABCB1-overexpressing drug-resistant mouse lymphoma $(12,13)$.

In 2012, through small molecule library screening, Sachlos et al (14) discovered that thioridazine exhibited antineoplastic pluripotent stem cell ability, without interfering with normal pluripotent stem cells. Furthermore, it reduced the function of leukemia stem cells while sparing the normal human repopulation cells, demonstrating thioridazine's specificity in targeting CSCs. As CSCs play a vital role in drug resistance, cancer relapse and metastasis, thioridazine may be an effective drug for cancer therapy by targeting CSCs. Since thioridazine has been safely used in the clinic for many years as an antipsychotic, it can be rapidly evaluated in the clinic as an anticancer drug. However, the antitumor effects of thioridazine on gastric cancer have never been disclosed, and to the best of our knowledge, the in vivo tumor inhibition capacity of thioridazine has only been reported in murine cancer cells (15). In the present study, we examined the cytotoxic effects of thioridazine on gastric cancer cell lines NCI-N87 and AGS. Thioridazine reduced the cell viability of gastric cancer cells and inhibited the colony formation of NCI-N87 and AGS cells. Thioridazine induced gastric cancer cell apoptosis dependent on caspases and through the mitochondrial pathway. Moreover, thioridazine pretreatment inhibited the growth of NCI-N87 cell-derived tumors. The present study demonstrated the anticancer effects of thioridazine in gastric cancer cells, and its ability to inhibit human gastric cancer cell tumor growth in vivo, suggesting thioridazine as a candidate for gastric cancer therapy.

\section{Materials and methods}

Cell lines and cell culture. Gastric cancer cell lines NCI-N87 and AGS were obtained from the Cell Bank of the Shanghai Institutes for Biological Sciences, Chinese Academy of Sciences (Shanghai, China). NCI-N87 cells were maintained in DMEM supplemented with $10 \%$ heat-inactivated fetal bovine serum (FBS), and AGS cells were cultured in F-12K with $10 \%$ FBS. Both types of cells were incubated at $37^{\circ} \mathrm{C}$ in an atmosphere with $5 \% \mathrm{CO}_{2}$.

Analysis of cell viability. The NCI-N87 and AGS cells were plated in 96-well plates at a density of $10^{4}$ cells/well, and were treated with thioridazine (Sigma) at indicated concentrations for $48 \mathrm{~h}$. After incubation with $0.5 \mathrm{mg} / \mathrm{ml} \mathrm{3-(4,5-dimethylthi-}$ azol-2-yl)-2,5-diphenyl tetrazolium bromide(MTT) (Beyotime Institute of Biotechnology, Haimen, China) for another $4 \mathrm{~h}$, the supernatant was discarded, and $100 \mu$ 1 DMSO (dimethyl sulfoxide) was added to dissolve the produced formazan crystals. The absorbance was measured on a microplate reader (Thermo Fisher Scientific) at a dual wavelength of $595 \mathrm{~nm}$ and $630 \mathrm{~nm}$. The difference in the outcome of $\mathrm{OD}_{595}$ and $\mathrm{OD}_{630}$ was considered as the measure of cell viability.

Observation of cytotoxicity. To directly observe the cytotoxic effects of thioridazine, NCI-N87 and AGS cells were seeded in 24-well plates and incubated with thioridazine at the indicated concentrations. Forty-eight hours post-treatment, the cells were directly observed using microscopy or stained with $2 \%$ crystal violet which was dissolved in $20 \%$ methanol solution.

Colony formation assay. The NCI-N87 cells were seeded into 6-well plates at 3,000 cells/well and treated with thioridazine at indicated concentrations after the cells recovered. The formed colonies were stained with $2 \%$ crystal violet 10 days later, washed and photographed. The AGS cells were seeded at 1,000 cells/well in 6-well plates, treated similarly to NCI-N87 cells, and were stained with crystal violet 6 days later.

Western blot assay. Western blot analysis was performed according to standard procedures. In brief, cells were trypsinized following treatment for the indicated times, lysed using RIPA lysis buffer and boiled in SDS-PAGE sample loading buffer (both from Beyotime Institute of Biotechnology). Total protein $(40 \mu \mathrm{g})$ was electrophoretically separated on 10 or $12 \%$ SDS-PAGE gel and transferred onto a polyvinylidene difluoride (PVDF) membrane. The PVDF membrane was blocked with milk, incubated with the primary antibodies and then with the secondary antibodies. Primary antibodies for caspase-9, caspase-8, caspase-3 and PARP were purchased from Santa Cruz Biotechnology (Santa Cruz, CA, USA) and GAPDH was from CoWin Bioscience Co. (Beijing, China). All the secondary antibodies were obtained from Santa Cruz Biotechnology. Subsequently, the protein on the membrane was revealed using ECL reagents (Pierce Biotechnology, Rockford, IL, USA).

Hoechst 33258 staining. The AGS cells were treated with thioridazine at indicated concentrations, and fixed with $4 \%$ paraformaldehyde (PFA) for $15 \mathrm{~min} 48 \mathrm{~h}$ post-treatment. The cells were then stained with Hoechst 33258 (Molecular Probes, Eugene, OR, USA) at $1 \mu \mathrm{g} / \mathrm{ml}$ for $1 \mathrm{~min}$, washed with PBS and photographed under a fluorescence microscope.

Cell cycle analysis. The NCI-N87 and AGS cells were trypsinized following treatment with thioridazine for $48 \mathrm{~h}$, and then resuspended in PBS and fixed with $70 \%$ ethanol overnight at $-20^{\circ} \mathrm{C}$. The fixed cells were centrifugated, washed with PBS and incubated with $20 \mu \mathrm{g} / \mathrm{ml}$ RNAase in a water bath at $37^{\circ} \mathrm{C}$ for $30 \mathrm{~min}$. After been stained with $50 \mu \mathrm{g} / \mathrm{ml}$ PI for another $30 \mathrm{~min}$, they were subjected to a fluorescence-activated cell sorting (FACS) instrument for analysis.

Detection of apoptosis. The NCI-87 and AGS cells were treated with thioridazine for $48 \mathrm{~h}$. Cells were trypsinized into single cells, washed with PBS and binding buffer, and then 
resuspended in $500 \mu \mathrm{l}$ binding buffer. Annexin V-FITC (5 $\mu \mathrm{l})$ and $10 \mu \mathrm{l}$ propidium iodide (PI, $20 \mu \mathrm{g} / \mathrm{ml}$ ) were subsequently added to the cells for incubation. Fifteen minutes later, the cells were washed with binding buffer and subjected to FACS for analysis.

Analysis of mitochondrial membrane potential alteration. Alteration in mitochondrial membrane potential is detected by JC-1 staining assay. Unimpaired cells retain high mitochondrial membrane potential, and JC-1 accumulates in the matrix of the mitochondria and propagates as aggregates, which emit red fluorescence in the presence of excitation. However, the mitochondrial membrane potential decreases as cells are impaired, and then JC-1 diffuses into the cytoplasm and forms monomers, which emit green fluorescence after excitation. The experiment was performed as follows.

The cells were seeded in 6-well plates at a density of $3 \times 10^{6}$ cells/well and treated with thioridazine for $48 \mathrm{~h}$. After been trypsinized, cells were resuspended in $500 \mu \mathrm{l}$ DMEM, and then mixed with $500 \mu \mathrm{l}$ JC-1 working solution and incubated for $20 \mathrm{~min}$ at $37^{\circ} \mathrm{C}$ in the dark. Afterwards, the cells were washed twice with JC-1 staining buffer, resuspended in $300 \mu \mathrm{l}$ JC-1 staining buffer and assessed by FACS.

Animal experiments. The animal experiments were performed according to the U.S. Public Health Service Policy on Humane Care and the Use of Laboratory Animals, and were also approved by the Institutional Animal Care and Use Committee. Four-week-old female BALB/c nude mice were purchased from Shanghai Laboratory Animal Center (SLAC; Shanghai, China). To test the ability of thioridazine to inhibit tumor growth, the NCI-N87 cells were incubated with DMSO or $5 \mu \mathrm{M}$ thioridazine for $24 \mathrm{~h}$. The treated cells were trypsinized and counted after been stained with trypan blue. Viable DMSO-pretreated $\left(1 \times 10^{6}\right)$ and thioridazine-pretreated NCI-N87 cells were subcutaneously injected into the left and right rear of mice, respectively. After visible tumors emerged, they were measured every three days. The volume was calculated as length $\mathrm{x}$ width $\mathrm{x}$ width/2.

Statistical analysis. Comparison of groups was analyzed by the Student's t-test. A p-value of less than 0.05 was considered to indicate a statistically significant difference.

\section{Results}

Thioridazine exhibits cytotoxicity in gastric cancer cells. To initially assess the anticancer effect of thioridazine on gastric cancer cells, MTT assay was performed on treated cells. Thioridazine reduced the cell viability of NCI-N87 and AGS cells in a concentration-dependent manner (Fig. 1A). Moreover, severe morphological alterations were observed in the majority of the cells treated with $15 \mu \mathrm{M}$ thioridazine (Fig. 1B). Results of the crystal violet staining clearly disclosed the cytotoxic effect of thioridazine on NCI-N87 and AGS cells (Fig. 1C). Furthermore, $5 \mu \mathrm{M}$ thioridazine, which showed a slight effect on the cell viability of NCI-N87 and AGS cells, markedly inhibited their colony formation ability (Fig. 1D). Thioridazine at a concentration of $2 \mu \mathrm{M}$ also exhibited a slight inhibitory effect on the colony formation of NCI-N87 and AGS cells.
Thioridazine induces gastric cancer cell apoptosis. Thioridazine was previously found to induce cervical and endometrial cancer apoptosis. To detect whether thioridazine induces gastric cancer cell apoptosis, Hoechst 333258 staining was carried out. Severe nuclear fragmentation was observed in the AGS cells following treatment with thioridazine at 10 and $15 \mu \mathrm{M}$, but not in the cells treated with DMSO or thioridazine at a low dosage (Fig. 2A). As the appearance of nuclear fragmentation indicates the occurrence of apoptosis, the cell cycle distribution was analyzed in the treated and untreated cells. The proportion of sub-G1 phase cells in the NCI-N87 and AGS cells increased as the dosage of thioridazine was increased (Fig. 2B). Further detection by Annexin V/PI double staining assay revealed that thioridazine treatment resulted in an increase in the percentage of Annexin V-positive cells in both the NCI-N87 and AGS cells (Fig. 2C). The results indicate the occurrence of apoptosis in the thioridazine-treated gastric cancer cells.

Thioridazine induces gastric cancer cell apoptosis dependent on the caspases. To further clarify the mechanism of thioridazine-induced apoptosis, expression of a number of apoptosis-related proteins was analyzed. PARP cleavage was observed in the presence of thioridazine, which further supported the occurrence of apoptosis. Precursors of caspase-9, caspase- 8 and caspase-3 were decreased in the NCI-N87 cells following the treatment of thioridazine for $48 \mathrm{~h}$. Moreover, downregulation of precursors of caspase- 9 , caspase- 8 and caspase- 3 was observed in the AGS cells treated with $15 \mu \mathrm{M}$ thioridazine for 24 or $48 \mathrm{~h}$ (Fig. 3A). These results indicate the involvement of caspases in thioridazine-induced apoptosis in a time- and dose-dependent manner. Furthermore, caspase inhibitor Z-VAD-FMK was able to reverse the cytotoxicity of thioridazine both in the NCI-N87 and AGS cells (Fig. 3B), suggesting that thioridazine induced gastric cancer cell apoptosis in a caspase-dependent manner.

Thioridazine induces cell apoptosis via the mitochondrial pathway. As caspase-9 is involved in the mitochondrial apoptosis pathway, a decreased level of caspase-9 precursor implied the breakdown of mitochondria in the thioridazine-induced apoptosis. JC-1 staining assay was then carried out to examine the alteration of mitochondria. The percentage of cells with loss of mitochondrial membrane potential increased from 21.7 to $40.2 \%$ in the NCI-N87 cells following treatment with $15 \mu \mathrm{M}$ thioridazine (Fig. 4A), and this proportion increased from 12.5 to $49.8 \%$ in AGS cells (Fig. 4B). The loss of mitochondrial membrane potential, along with decreased caspase-9 (Fig. 3A), suggest that thioridazine induced gastric cancer cell death via the mitochondrial apoptosis pathway.

Thioridazine inhibits tumor growth in vivo. To test the in vivo activity of thioridazine on gastric cancer cells, NCI-N87 cells were pretreated with DMSO or $5 \mu \mathrm{M}$ thioridazine for $24 \mathrm{~h}$, and the cells were then injected subcutaneously into the nude mice at the left or right rear to compare their tumor formation ability. Thioridazine $(5 \mu \mathrm{M})$ elicited little change in the cell viability of NCI-N87 cells (Fig. 1A); however, pretreatment with $5 \mu \mathrm{M}$ thioridazine inhibited the growth of NCI-N87 cell-derived tumors (Fig. 5A), and this was consistent with 
A

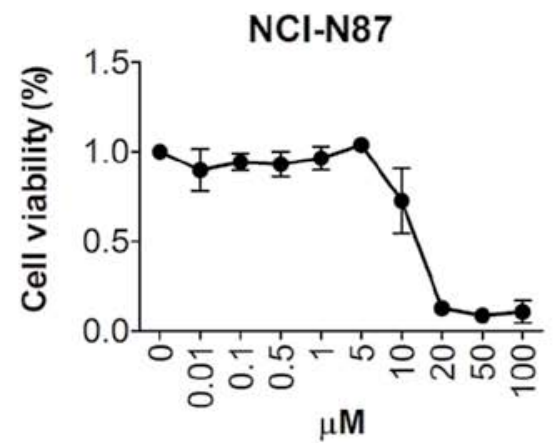

AGS

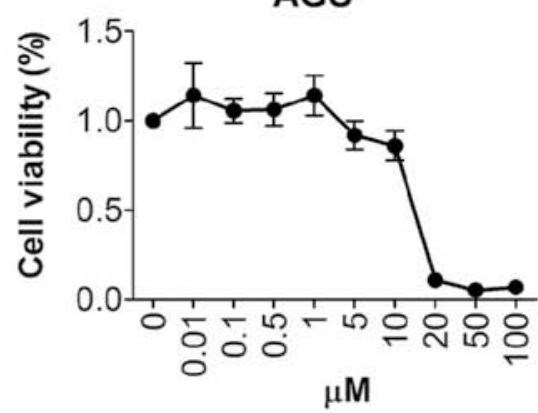

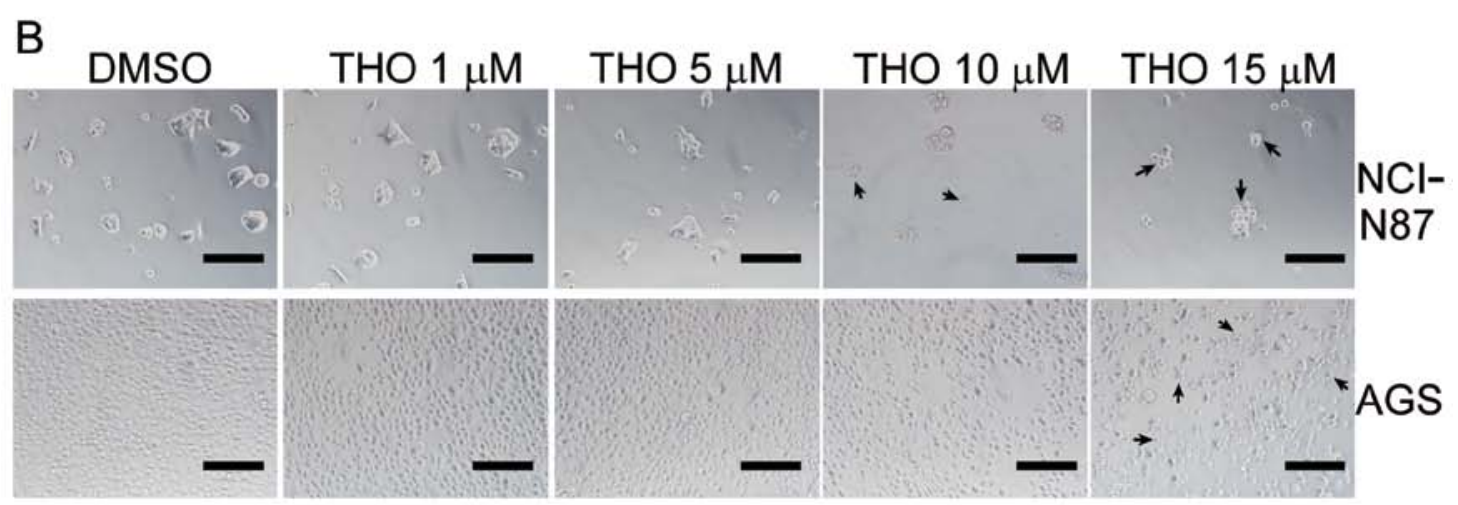

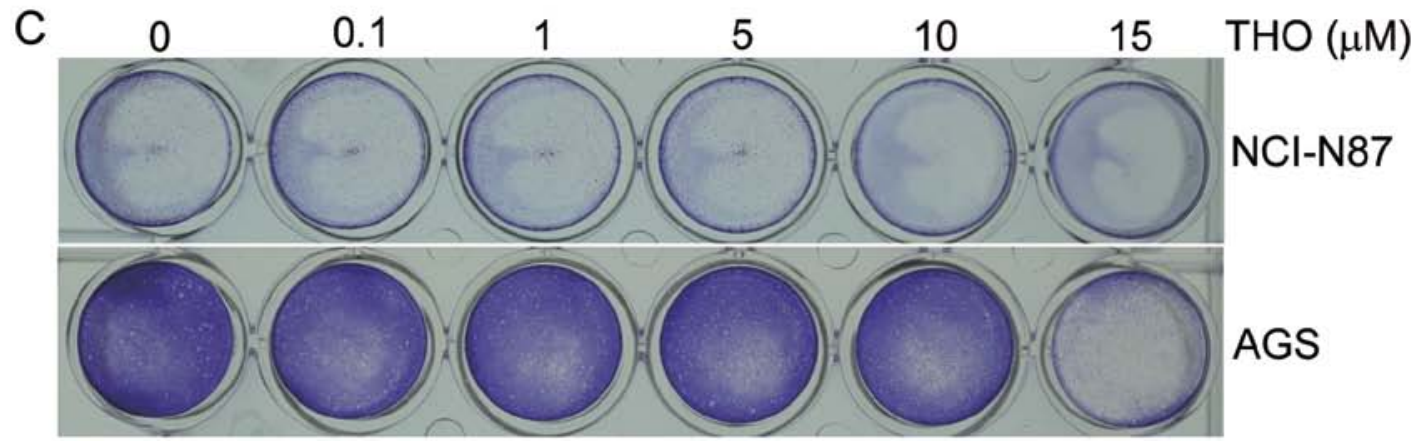

D

$\mathrm{NCl}-\mathrm{N} 87$

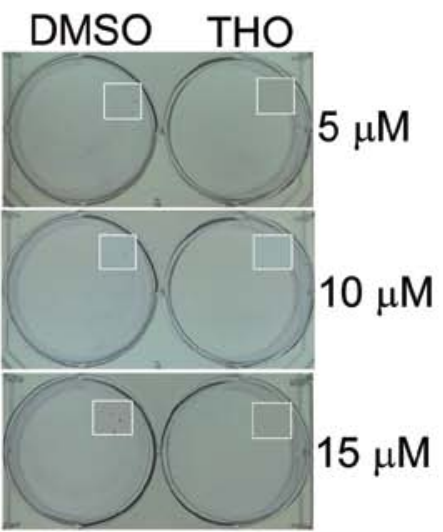

AGS

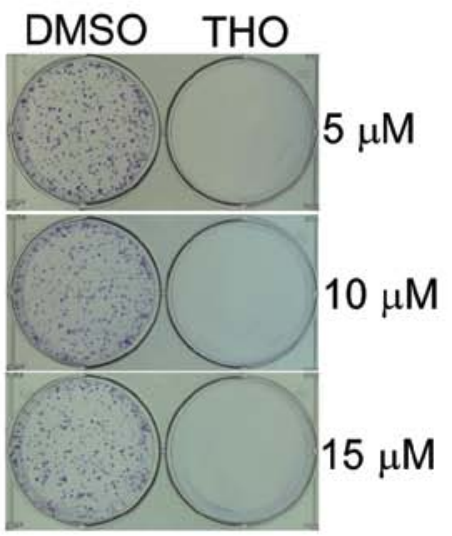

Figure 1. Thioridazine exhibits cytotoxic effects in gastric cancer cells. (A) Assessment of the cell viability of NCI-N87 and AGS cells following treatment with thioridazine at the indicated concentrations. (B) Morphology of thioridazine-treated NCI-N87 and AGS cells. Scale bar, $500 \mu$ m. Arrows indicate the cells with severe morphological alteration. (C) Crystal violet staining of cells treated with thioridazine at the indicated dosages. (D) Detection of colony formation of thioridazine-treated NCI-N87 and AGS cells. Amplified regions are shown in the square frames, respectively. THO, thioridazine.

thioridazine's ability to inhibit colony formation (Fig. 1D). Obviously smaller tumors were observed at the right rear region of the mice which were derived from the thioridazine- pretreated cells, and the resected tumors exhibited a similar result (Fig. 5B and C). Moreover, the weight of the tumors derived from the thioridazine-pretreated cells was lower than 
A

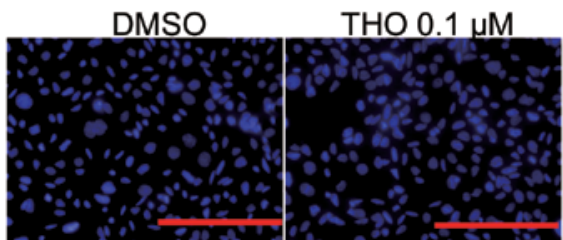
THO $1 \mu \mathrm{M}$

THO $5 \mu \mathrm{M}$

THO $10 \mu \mathrm{M}$

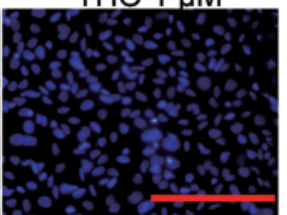

THO $15 \mu \mathrm{M}$
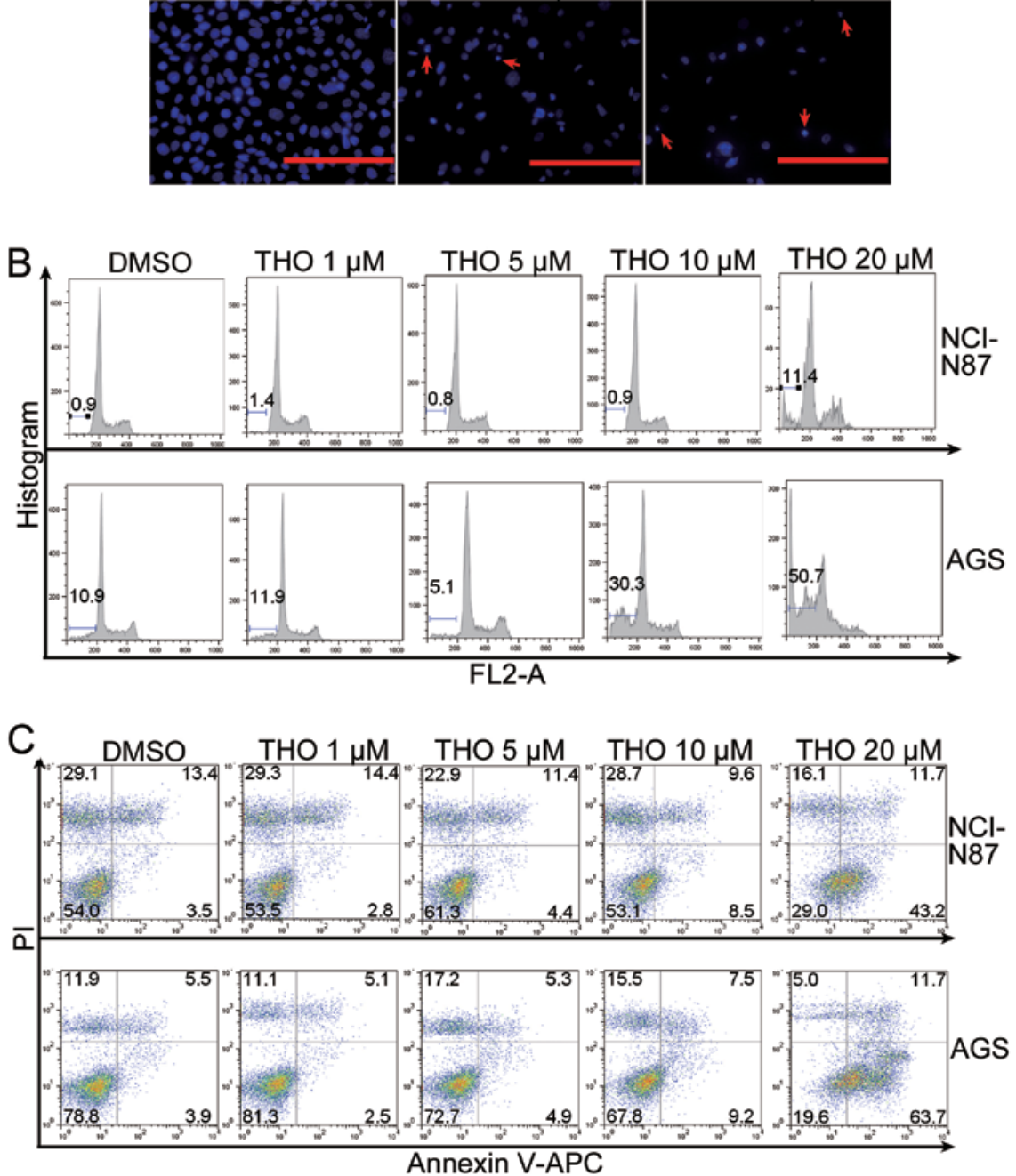

Figure 2. Thioridazine induces gastric cancer cell apoptosis. (A) Observation of nuclear morphology of thioridazine (THO)-treated cells. Scale bar, 200 $\mu \mathrm{m}$. Arrows indicate fragmented nuclei. Amplified images of fragmented nuclei are shown at the upper right panel of the figures. (B) Detection of cell cycle distribution of NCI-N87 and AGS cells following thioridazine treatment. (C) Annexin V/PI double staining analysis of thioridazine-treated NCI-N87 and AGS cells.

the tumors derived from the cells pretreated with DMSO (Fig. 5D).

\section{Discussion}

Thioridazine has been reported to have antiproliferative capacity in cancer cells, and is capable of inducing cancer cell apoptosis in various types of cancers (3-6). However, the cytotoxic effect of thioridazine in gastric cancer cells has never been reported. The present study revealed that thioridazine reduced the cell viability of gastric cancer cells, inhibited gastric cancer cell colony formation, and induced gastric cancer cell apoptosis via the mitochondrial pathway. These results indicate that thioridazine possesses anti-gastric cancer ability. Further experiments disclosed that thioridazine pretreatment inhibited the in vivo growth of tumors derived from NCI-N87 cells. To the best of our knowledge, to date, there is no research concerning the in vivo inhibition of human tumor growth by thioridazine, but only research disclosing its ability to inhibit murine tumor growth (15). The present study further demonstrated the in vivo tumor inhibitory ability of thioridazine and disclosed its cytotoxic effect on gastric cancer cells, suggesting thioridazine as a candidate drug for gastric cancer therapy.

Previous studies have disclosed the ability of thioridazine to reversie chemoresistance, and thioridazine was able to prevent the exclusion of small molecules from cancer cells $(10,12,16)$. This may explain the anti-CSC ability of thioridazine that was discovered by small molecule library screening (17). Thioridazine was found to have an influence 
A

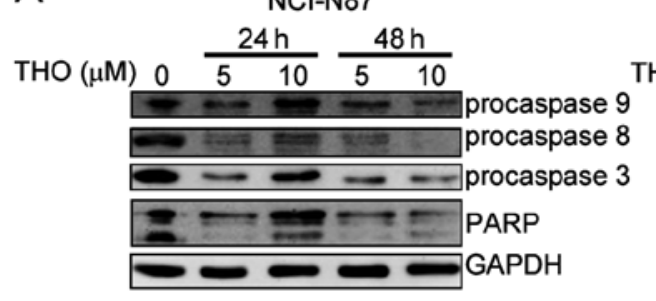

B

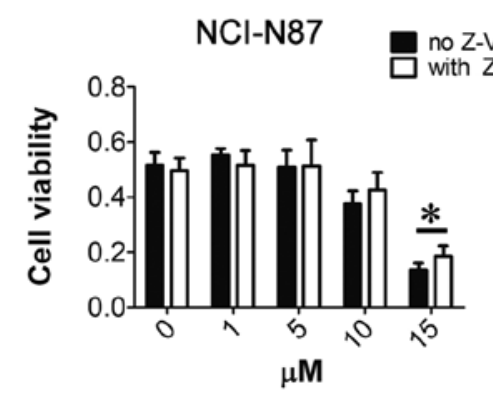

AGS

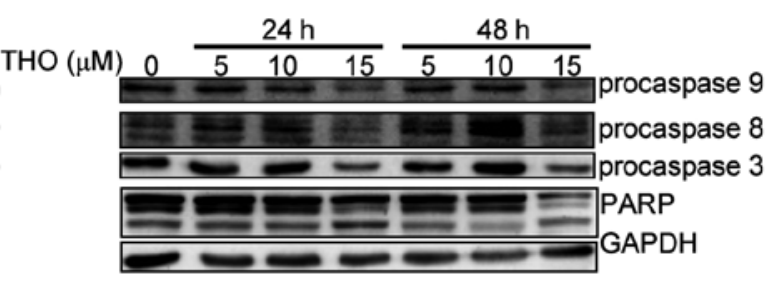

AGS

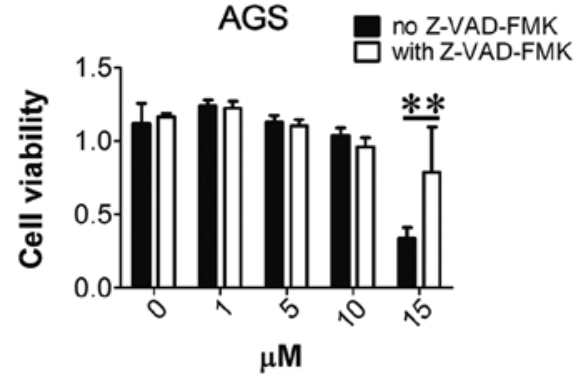

Figure 3. Thioridazine induces gastric cancer cell apoptosis dependent on the caspases. (A) Western blot analysis of the alteration of apoptosis-associated proteins. (B) Caspase inhibitor Z-VAD-FMK reversed thioridazine-induced cell death. ${ }^{*} \mathrm{P}<0.05,{ }^{* *} \mathrm{P}<0.01$. THO, thioridazine.

A

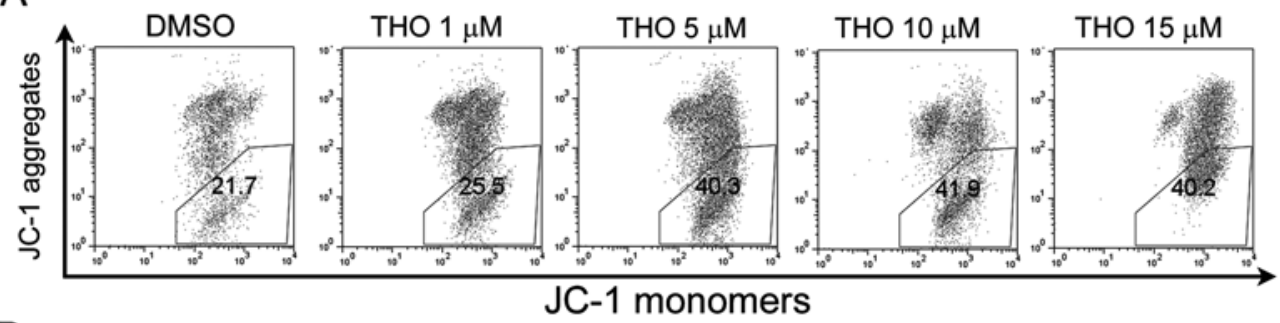

B

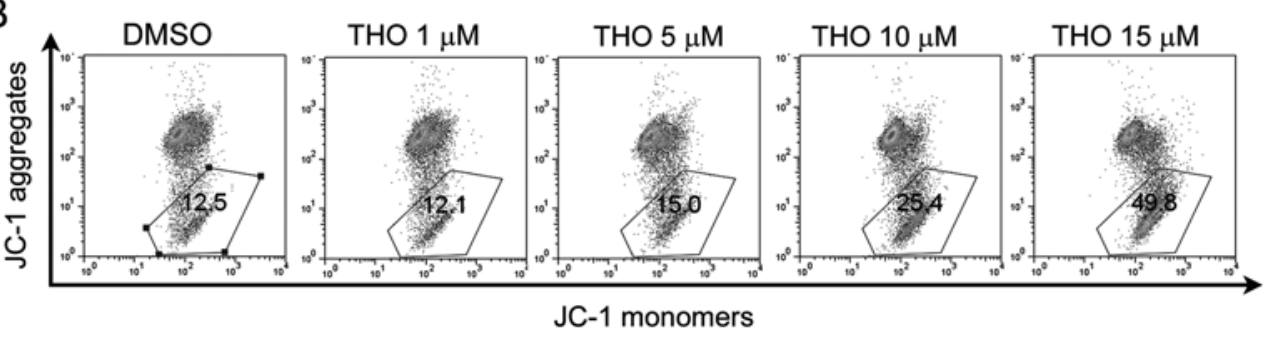

Figure 4. Thioridazine induces cell apoptosis via the mitochondrial pathway. Determination of the loss of mitochondrial membrane potential in (A) NCI-N87 and (B) AGS cells by JC-1 staining. Values in the trapeziform regions indicate the proportion of cells with loss of mitochondrial membrane potential. THO, thioridazine.

on cyclin and cyclin-dependent kinase (CDK) $(3,7)$, which are correlated with the cell cycle and cell growth. Moreover, thioridazine inhibited the PI3K/Akt pathway $(3,7)$, which plays a critical role in CSCs (18). Moreover, DRD2, which was antagonized by thioridazine, specifically regulated Wnt and Akt signaling (17). Thus, thioridazine may elicit its anti-CSC capacity by preventing the exclusion of small molecules out of CSCs and by inhibiting the Wnt and PI3K/Akt pathways in CSCs. However, this needs to be confimred by further experiments. Thioridazine was shown to have anti-gastric cancer ability both in vitro and in vivo in the present study, yet whether it has an effect on gastric CSCs was not elucidated. Studies are needed to isolate gastric CSCs (19) and to assess the effects of thioridazine on gastric CSCs.
Thioridazine was previously found to reverse the chemoresistance of cancer cells, and a significant therapeutic outcome in combination with verapamil was noted (11). Thus, thioridazine may be considered as an adjuvant in combination with chemotherapeutic drugs for cancer therapy. Moreover, it may also be utilized to treat cancer cells together with viruses. Thioridazine is an antagonist of the dopamine receptor D2 family proteins, and analogues of thioridazine have also exhibited antitumor effects (15), implying the involvement of the dopamine receptor signaling pathway in cancer. Dopamine receptor family proteins have been reported to be correlated with cancer therapy $(20,21)$. Thioridazine may elicit tumor growth inhibition in a dopamine receptor-dependent and -independent manner. Negative relationship of the expression 


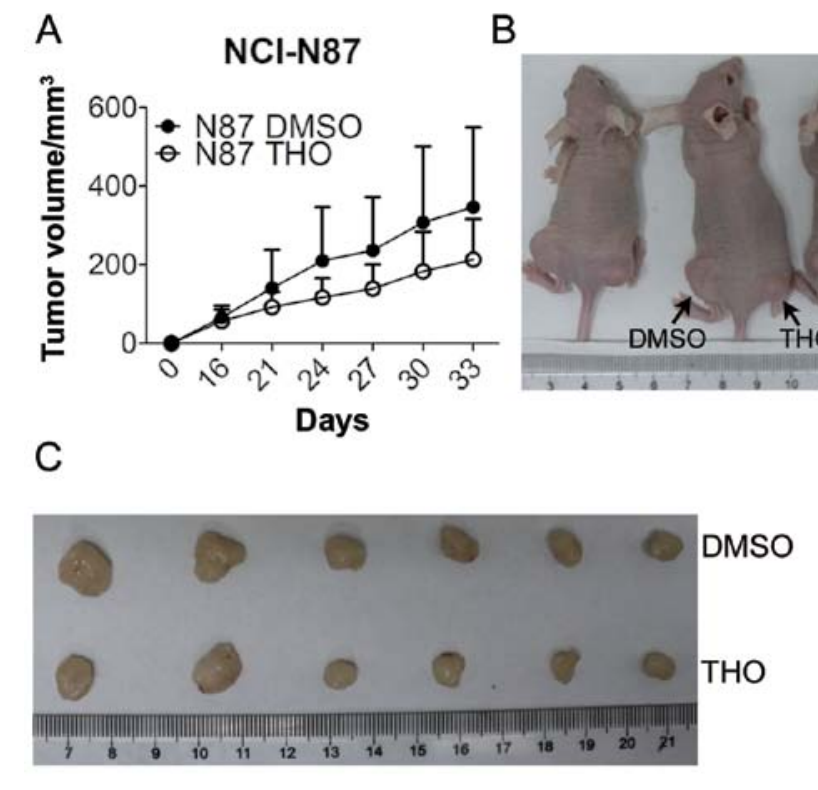

B

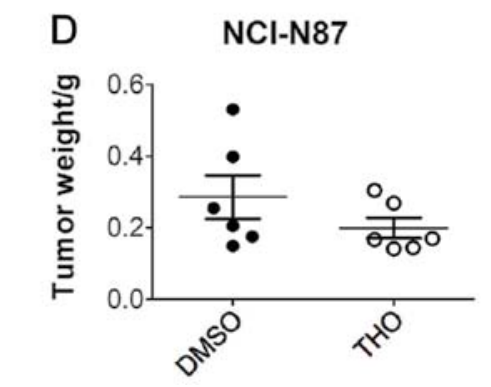

Figure 5. Thioridazine inhibits tumor growth in vivo. (A) Growth curve of tumors derived from NCI-N87 cells pretreated with DMSO or $5 \mu$ M thioridazine. (B) Comparison of tumors derived from cells pretreated with DMSO or thioridazine in nude mice on day 33. (C) Morphology of tumors with different treatments on day 33. (D) Measurement of tumor weights with indicated treatments on day 33. THO, thioridazine.

of DRD2 family protein and the prognosis of leukemia was revealed, and other antagonists of the DRD2 family were found to exhibit an effect against CSCs as well (17). These results suggest that blocking of DRD2 family proteins may inhibit the growth of cancer cells, even CSCs. Development of specific small molecules that target or knock down DRD2 family proteins may result in a favorable outcome. Overexpressing shRNA targeting DRD2 family proteins by oncolytic viruses, particularly specific tumor targeting vectors $(22,23)$, may exhibit a great antitumor effect. However, selective agonist of DRD2 family proteins induced lung cancer cell apoptosis $(24,25)$, and this was contrary with the effect of the function of DRD2 family proteins in leukemia (17). Thus, the strategy of targeting DRD2 needs to be considered in relation to different types of cancers.

To overcome gastric cancer, it is vital to combine prevention as well as effective therapy. Habits including the eating of fresh food, avoiding the consumption of cured food and smoking, detecting Helicobacter pylori infection and screening will reduce the incidence of gastric cancer (2). However, in regards to patients diagnosed with gastric cancer or even advanced gastric cancer, treatment using effective drugs is vital. In the present study, thioridazine exhibited favorable anti-gastric cancer effects in vitro and in vivo, suggesting its use as a promising candidate drug for gastric cancer therapy. Furthermore, its combination with other therapeutic drugs may exhibit favorable outcome in gastric cancer therapy.

\section{Acknowledgements}

The present study was supported by the National Natural Science Foundation of China (nos. 81172026, 81272402, 81301816 and 81172029), the Foundation of Shanghai Outstanding Academic Leaders (no. 11XD1403800), the
National High Technology Research and Development Program (863 Program) (no. 2012AA022606), the Postdoctoral Research Foundation of China (no. 2012M511107), the Foundation for Interdisciplinary Research of Shanghai Jiao Tong University (no. YG2011ZD07), the Shanghai Science and Technology Commission Inter-governmental International Cooperation Project (12410705900), the Shanghai Science and Technology Commission MedicalGuiding Project (12401905800), the Program for Changjiang Scholars and the Post-doctoral Research Program of Shanghai (no. 12R21415300).

\section{References}

1. Parkin DM, Bray F, Ferlay J and Pisani P: Global cancer statistics, 2002. CA Cancer J Clin 55: 74-108, 2005.

2. Jemal A, Bray F, Center MM, Ferlay J, Ward E and Forman D: Global cancer statistics. CA Cancer J Clin 61: 69-90, 2011.

3. Kang S, Dong SM, Kim BR, Park MS, Trink B, Byun HJ and Rho SB: Thioridazine induces apoptosis by targeting the PI3K/ Akt/mTOR pathway in cervical and endometrial cancer cells. Apoptosis 17: 989-997, 2012.

4. Strobl JS, Kirkwood KL, Lantz TK, Lewine MA, Peterson VA and Worley JF III: Inhibition of human breast cancer cell proliferation in tissue culture by the neuroleptic agents pimozide and thioridazine. Cancer Res 50: 5399-5405, 1990.

5. Gil-Ad I, Shtaif B, Levkovitz Y, Dayag M, Zeldich E and Weizman A: Characterization of phenothiazine-induced apoptosis in neuroblastoma and glioma cell lines: clinical relevance and possible application for brain-derived tumors. J Mol Neurosci 22: 189-198, 2004.

6. Zhelev Z, Ohba H, Bakalova R, Hadjimitova V, Ishikawa M, Shinohara $\mathrm{Y}$ and Baba Y: Phenothiazines suppress proliferation and induce apoptosis in cultured leukemic cells without any influence on the viability of normal lymphocytes. Phenothiazines and leukemia. Cancer Chemother Pharmacol 53: 267-275, 2004.

7. Rho SB, Kim BR and Kang S: A gene signature-based approach identifies thioridazine as an inhibitor of phosphatidylinositol3'-kinase (PI3K)/AKT pathway in ovarian cancer cells. Gynecol Oncol 120: 121-127, 2011. 
8. Byun HJ, Lee JH, Kim BR, Kang S, Dong SM, Park MS, Lee SH, Park SH and Rho SB: Anti-angiogenic effects of thioridazine involving the FAK-mTOR pathway. Microvasc Res 84: 227-234, 2012.

9. Ramu A, Spanier R, Rahamimoff $\mathrm{H}$ and Fuks Z: Restoration of doxorubicin responsiveness in doxorubicin-resistant P388 murine leukaemia cells. Br J Cancer 50: 501-507, 1984.

10. Akiyama S, Shiraishi N, Kuratomi Y, Nakagawa M and Kuwano M: Circumvention of multiple-drug resistance in human cancer cells by thioridazine, trifluoperazine, and chlorpromazine. J Natl Cancer Inst 76: 839-844, 1986.

11. Castaing M, Loiseau A and Cornish-Bowden A: Synergy between verapamil and other multidrug-resistance modulators in model membranes. J Biosci 32: 737-746, 2007.

12. Kamiwatari M, Nagata Y, Kikuchi H, Yoshimura A, Sumizawa T, Shudo N, Sakoda R, Seto K and Akiyama S: Correlation between reversing of multidrug resistance and inhibiting of $\left[{ }^{3} \mathrm{H}\right]$ azidopine photolabeling of P-glycoprotein by newly synthesized dihydropyridine analogues in a human cell line. Cancer Res 49 3190-3195, 1989.

13. Spengler G, Molnar J, Viveiros M and Amaral L: Thioridazine induces apoptosis of multidrug-resistant mouse lymphoma cells transfected with the human ABCB1 and inhibits the expression of P-glycoprotein. Anticancer Res 31: 4201-4205, 2011.

14. Sachlos E, Risueno RM, Laronde S, et al: Identification of drugs including a dopamine receptor antagonist that selectively target cancer stem cells. Cell 149: 1284-1297, 2012.

15. Gil-Ad I, Shtaif B, Levkovitz Y, Nordenberg J, Taler M, Korov I and Weizman A: Phenothiazines induce apoptosis in a B16 mouse melanoma cell line and attenuate in vivo melanoma tumor growth. Oncol Rep 15: 107-112, 2006

16. Efferth $\mathrm{T}$ and Volm M: Reversal of doxorubicin-resistance in sarcoma 180 tumor cells by inhibition of different resistance mechanisms. Cancer Lett 70: 197-202, 1993.
17. Sutton LP and Rushlow WJ: The dopamine D2 receptor regulates Akt and GSK-3 via Dvl-3. Int J Neuropsychopharmacol 15: 965-979, 2012

18. Dubrovska A, Kim S, Salamone RJ, Walker JR, Maira SM, Garcia-Echeverria C, Schultz PG and Reddy VA: The role of PTEN/Akt/PI3K signaling in the maintenance and viability of prostate cancer stem-like cell populations. Proc Natl Acad Sci USA 106: 268-273, 2009.

19. Takaishi S, Okumura T, Tu S, Wang SS, Shibata W, Vigneshwaran R, Gordon SA, Shimada Y and Wang TC: Identification of gastric cancer stem cells using the cell surface marker CD44. Stem Cells 27: 1006-1020, 2009.

20. Gabalec F, Beranek M, Netuka D, Masopust V, Nahlovsky J, Cesak T, Marek J and Cap J: Dopamine 2 receptor expression in various pathological types of clinically non-functioning pituitary adenomas. Pituitary 15: 222-226, 2012.

21. Gatto F and Hofland LJ: The role of somatostatin and dopamine D2 receptors in endocrine tumors. Endocr Relat Cancer 18: R233-R251, 2011.

22. Xu HN, Huang WD, Cai Y, et al: HCCS1-armed, quadrupleregulated oncolytic adenovirus specific for liver cancer as a cancer targeting gene-viro-therapy strategy. Mol Cancer 10: 133, 2011.

23. Ding M, Cao X, Xu HN, et al: Prostate cancer-specific and potent antitumor effect of a $D D 3$-controlled oncolytic virus harboring the PTEN gene. PLoS One 7: e35153, 2012.

24. Sheikhpour M, Ahangari G, Sadeghizadeh M and Deezagi A: A novel report of apoptosis in human lung carcinoma cells using selective agonist of D2-like dopamine receptors: a new approach for the treatment of human non-small cell lung cancer. Int $J$ Immunopathol Pharmacol 26: 393-402, 2013.

25. Senogles SE: D2 dopamine receptor-mediated antiproliferation in a small cell lung cancer cell line, NCI-H69. Anticancer Drugs 18: 801-807, 2007. 